\title{
COSMOLOGICAL IMPLICATIONS OF THE VERY HIGH REDSHIFT GRB 050904
}

\author{
P. A. Price, ${ }^{1}$ L. L. Cowie, ${ }^{1}$ T. Minezaki, ${ }^{2}$ B. P. Schmidt, ${ }^{3}$ A. Songaila, ${ }^{1}$ and Y. Yoshii ${ }^{2}$ \\ Received 2005 September 22; accepted 2006 March 21
}

\begin{abstract}
We report near-simultaneous multicolor (RIYJHK) observations made with the MAGNUM $2 \mathrm{~m}$ telescope of the gamma-ray burst GRB 050904 detected by the Swift satellite. The spectral energy distribution shows a very large break between the $I$ and $J$ bands. Using intergalactic transmissions measured from high-redshift quasars, we show that the observations place a $95 \%$ confidence lower limit of $z=6.18$ on the object, consistent with a later measured spectroscopic redshift of 6.29 obtained by Kawai et al. with the Subaru telescope. We show that the break strength in the $R$ and $I$ bands is consistent with that measured in the quasars. Finally, we consider the implications for the star formation history at high redshift.
\end{abstract}

Subject headings: cosmology: observations — galaxies: distances and redshifts — gamma rays: bursts

Online material: color figure

\section{INTRODUCTION}

Identifying the epoch of reionization remains a prime objective in modern cosmology. Quasars at the highest redshifts $z>$ 6.1, of which five are known (Fan et al. 2001, 2003, 2004, 2005), show a complete absorption trough blueward of the redshifted Ly $\alpha$ line from scattering by neutral hydrogen in the intergalactic medium (Becker et al. 2001), the so-called Gunn-Peterson effect (Gunn \& Peterson 1965). However, because of the extreme sensitivity of the attenuation to the neutral fraction of $\mathrm{H} \mathrm{I}$, it is only possible to deduce that $z \sim 6$ signals the end of the epoch of reionization (or even the end of the most recent epoch of reionization; e.g., Wyithe \& Loeb 2003).

One way of robustly determining the actual epoch of reionization is to measure the luminosity function of the Ly $\alpha$ flux of high-redshift galaxies, which will be attenuated by the damping wing of the Gunn-Peterson trough in a less severe manner than the continuum flux blueward of $\operatorname{Ly} \alpha$. Several searches for Ly $\alpha$ emitters (LAEs) at $z \sim 6.5$ have been made, yielding a (currently published) total of 13 that have been spectroscopically confirmed (Hu et al. 2002; Kodaira et al. 2003 [two]; Rhoads et al. 2004; Kurk et al. 2004; Stern et al. 2005; Taniguchi et al. 2005 [seven new]). These sources show little evolution from analagous populations at $z \sim 5.7$ (Stern et al. 2005), perhaps indicating that we have not yet reached the epoch of reionization. Studies using color break samples show a drop in the star formation rate by about a factor of 5 at $z \sim 6$ from the peak at lower redshifts (Bunker et al. 2004; Bouwens et al. 2006), while the Ly $\alpha$ surveys appear to show a flatter evolution (Hu \& Cowie 2006).

The color break searches are restricted to the small deep fields observed with the Hubble Space Telescope, while the searches for LAEs are intensive, requiring large investments of premier ground-based facilities, and they select only the most luminous LAEs. These surveys may therefore be biased toward detecting the more vigorously star-forming galaxies that produce large Strömgren spheres which allow the Ly $\alpha$ flux to leak out. This

\footnotetext{
1 Institute for Astronomy, University of Hawaii, 2680 Woodlawn Drive, Honolulu, HI 96822; price@ifa.hawaii.edu, cowie@ifa.hawaii.edu, acowie@ifa hawaii.edu.

2 Institute of Astronomy, University of Tokyo, Mitaka, Tokyo 181-0015, Japan; minezaki@mtk.ioa.s.u-tokyo.ac.jp, yoshii@mtk.ioa.s.u-tokyo.ac.jp.

3 Research School of Astronomy and Astrophysics, Australian National University, Cotter Road, Weston, ACT 2611, Australia; brian@mso.anu.edu.au.
}

means that the measurement of the epoch of reionization will be biased toward higher redshifts. To address this bias, we require a means of homogeneously identifying more modest star-forming galaxies at high redshift; this is what gamma-ray bursts (GRBs) may provide.

The luminosity function of the optical afterglows of GRBs extends as bright as an absolute magnitude of $M_{R} \approx-31.5 \mathrm{mag}$ at $1 \mathrm{hr}$ after the GRB in the rest frame, and probably even brighter at earlier epochs. Because of this extreme luminosity, they can be detected to great distances and therefore provide an exciting way to find very high redshift galaxies beyond the current upper limits of $z \sim 7$ and to map the star formation history at these extreme redshifts in a way which, if not itself unbiased, is at least independent of the properties of the underlying galaxies (Lamb \& Reichart 2000).

Indeed, it was widely expected that the advent of the Swift satellite would produce a large rate of return of very high redshift GRBs (e.g., Bromm \& Loeb 2002). While the predictions appear, in hindsight, to have been somewhat optimistic, the sensitive Swift mission is detecting GRBs at a higher mean redshift of $z \sim 2$ (Berger et al. 2005c) than previous missions such as HETE-2, BeppoSAX, and the Interplanetary Network. While this may make the afterglows more difficult to identify, it also gave hope that Swift would find sources beyond the most distant known GRB ( $z=4.5$; Andersen et al. 2000). The burst GRB 050904 discussed in this paper does just that, pushing the redshift limit for GRBs beyond $z=6$. Hopefully it is just the first of many such detections stretching to still higher redshifts.

High-redshift GRBs are easy to distinguish with coordinated optical and near-infrared (NIR) observations, since the GunnPeterson effect drastically attenuates flux in the optical bands. The mean transmissions as a function of redshift based on quasar observations to $z=6.4$ are tabulated in Songaila (2004), and these can be used to obtain the redshift of a GRB from its colors. However, Swift's Ultraviolet and Optical Telescope (UVOT) is limited to observations at wavelengths bluer than about $6500 \AA$. For this reason, optical afterglows of GRBs at very high redshifts $(z>6)$ cannot be detected by the UVOT on Swift, and hence their properties must be characterized using ground-based NIR observations.

GRB 050904 triggered the Burst Alert Telescope (BAT) on Swift at 1:51 UTC on 2005 September 4 and was rapidly localized (Cummings et al. 2005a). Fox \& Cenko (2005) undertook 
$\mathbf{R}$
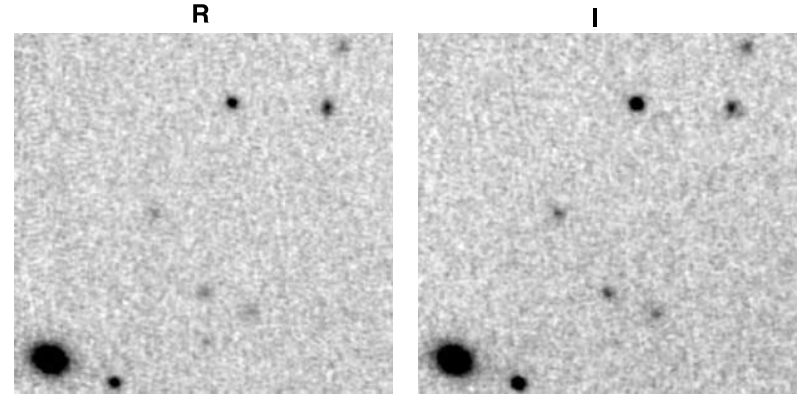

$\mathbf{Y}$

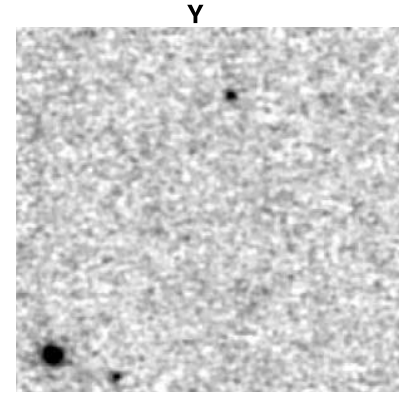

H
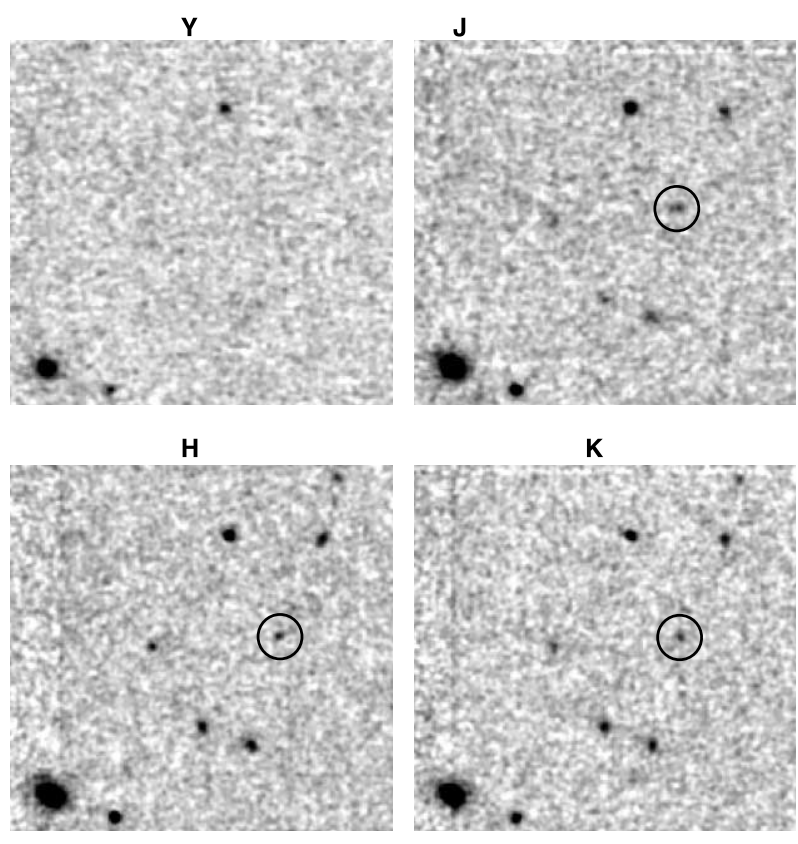

K

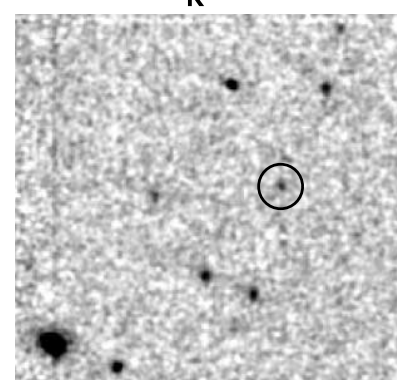

FIG. 1.- Images of GRB 050904 (circled object) in the RIYJHK bands, respectively. Each image is 1.2 on a side. The detector response is poor at $Y$, and the failure to detect the afterglow at this wavelength is not significant. [See the electronic edition of the Journal for a color version of this figure.]

optical observations in the optical $R$ and $i^{\prime}$ bands starting about $3.5 \mathrm{hr}$ after the GRB but did not identify any afterglow candidate to reasonable limiting magnitudes. The subsequent identification of a bright afterglow in the NIR $J$ band led to the interpretation that this was a GRB at very high redshift (Haislip et al. 2005).

Observations of the afterglow with the MAGNUM telescope began about $12 \mathrm{hr}$ after the GRB $(\S 2)$. These observations allow us to generate a spectral energy distribution for the source at that time which yields a strong lower limit on the redshift of $z=$ 6.18. This is consistent with the photometric redshifts reported by Haislip et al. (2005) and Tagliaferri et al. (2005), and the spectroscopic redshift of $z=6.295 \pm 0.002$ measured by Kawai et al. (2006). Furthermore, the limits on the break strengths $R-J$ and $I-J$ are consistent with the object lying at the spectrosopic redshift $(\S 3)$. We describe these observations in the present paper and briefly speculate on the implications for the star formation history of the universe $(\S 4)$.

\section{MAGNUM OBSERVATIONS OF GRB 050904}

MAGNUM (Multicolor Active Galactic NUclei Monitoring) is a $2 \mathrm{~m}$ telescope on Haleakala built by the Research Center for the Early universe (RESCEU) at the University of Tokyo and used to study AGN variability (Yoshii 2002). In order to optimize the efficiency of the monitoring observations, the telescope

TABLE 1

Measured Fluxes from MAGNUM

Observations in Multiple Bands

\begin{tabular}{cr}
\hline Band & $\begin{array}{c}\text { Flux } \\
(\mu \mathrm{Jy})\end{array}$ \\
\hline $\mathrm{n}$ & $-1.04 \pm 0.75$ \\
& $0.4 \pm 1.2$ \\
& $47.9 \pm 6.2$ \\
& $30.0 \pm 8.9$ \\
\hline
\end{tabular}

NoTES.- The observations were made at a mean epoch of 2005 September 4.60 and may be treated as simultaneous; any correction to the fluxes for the decay of the afterglow over the course of the observations would be smaller than the measurement errors $(0.15 \mathrm{mag}$, using a temporal decay index of 1.2 ; Haislip et al. 2005). These measurements are not corrected for the relatively small foreground Milky Way extinction. The $R$ - and $I$-band measurements are consistent with no detections to $3 \sigma$ upper limits on the fluxes of 2.3 and $3.6 \mu \mathrm{Jy}$, respectively.

is operated in a robotic mode using queue scheduling. GRB observations can be performed as soon as a notification is received by inserting the target and overriding the queue.

MAGNUM's principal instrumentation is the Multicolor Imaging Photometer (MIP; Kobayashi et al. 1998), a dualbeam optical/NIR camera that covers a 1.'5 square field in the $U B V R I Y J H K_{S} K L^{\prime}$ bands (although $U$ observations are difficult, and $L^{\prime}$ infeasible). The instrument, mounted at the Cassegrain focus, uses an internal beam splitter to send the short-wavelength light to a 1024 pixel square thinned CCD (although the entire CCD is not illuminated), and the long wavelengths to a 256 pixel square InSb array.

Because the limited field of view of the MIP makes it impractical to observe the $4^{\prime}$ localizations from the Swift BAT, we target afterglows discovered by the X-Ray Telescope (XRT) or ground-based follow-up observations in order to characterize their spectral flux distribution, and so attempt to determine a photometric redshift from the Ly $\alpha$ absorption at high redshift. We use a preplanned sequence of four observations, each consisting of nine individual minute-long exposures with a box dither pattern of $10^{\prime \prime}$ steps: RIRI in the optical and HKJY in the NIR. The final images provide an accurate and nearly simultaneous spectral energy distribution for the object. The entire sequence takes about $84 \mathrm{~min}-$ utes, with an additional 5 minutes before commencing the GRB observations in order to correct the pointing and focus after the slew.

We observed GRB 050904 in this manner between 13:57 and 15:07 UTC on 2005 September 4, or about 0.51 days after the GRB. The final combined images are shown in Figure 1. Because the only 2MASS source in the field is a galaxy, the images could not be immediately photometrically calibrated. We obtained observations on subsequent nights to obtain $I J K$ calibrations of two stars in the field. The $H$ band was calibrated from the 2MASS galaxy by using a large aperture to measure the entire flux of the galaxy and applying a measured aperture correction to obtain the flux of the afterglow. The $R$ band was calibrated using SDSS magnitudes for a star in the field and applying the appropriate transformation (Smith et al. 2002). The uncertainties in these two nonideal calibration methodologies do not strongly affect our results, since the $R$ and $H$ measurements are less important than the IJK measurements. The fluxes in $\mu \mathrm{Jy}$ with $1 \sigma$ errors are summarized in Table 1. These fluxes are measured in matched apertures corrected 


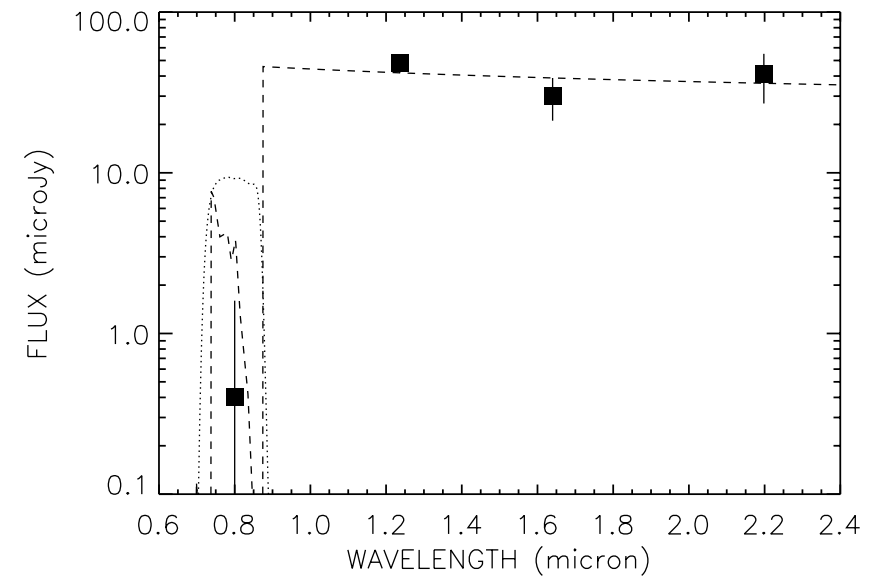

FIG. 2.-Spectral flux distribution of GRB 050904 from the MAGNUM observations is shown by the solid squares with $1 \sigma$ error bars. The dotted line shows the position of the $I$ filter. The dashed line shows the expected SFD of the object at a redshift of $z=6.18$ (our $2 \sigma$ lower limit on the redshift), based on a power-law fit to the longer $(J H K)$ wavelengths, modulated by the measured transmission of the intergalactic medium below the redshifted Ly $\alpha$ position, computed using the measurements of Songaila (2004).

for the image quality, with radii ranging from 1".4 to 1 ". 7 . The $Y$-band observations are not particularly sensitive and so are not included in our analysis. Since no source is apparent in the $R I$ bands, we measured the flux in the image at the position of the afterglow. Errors were taken as the rms from zero of the distribution offlux in apertures randomly distributed over empty background regions.

The difference between the $J$-band magnitude reported here and that obtained by UKIRT at a slightly earlier epoch, combined with the unusual behavior in the $Z$ band at about this epoch reported by Haislip et al. (2005), likely indicates that the source was somewhat variable during the period of these observations. Such short-timescale variability has been detected in other GRB optical afterglows, with GRB 030329 a notable example (Lipkin et al. 2004). This serves to demonstrate the need for simultaneous or near-simultaneous multicolor imaging, such as MAGNUM provides.

\section{REDSHIFT LIMITS AND THE BREAK STRENGTH}

We show the measured spectral flux distribution (SFD) of the source in Figure 2. There is a substantial break at the $I$ band that

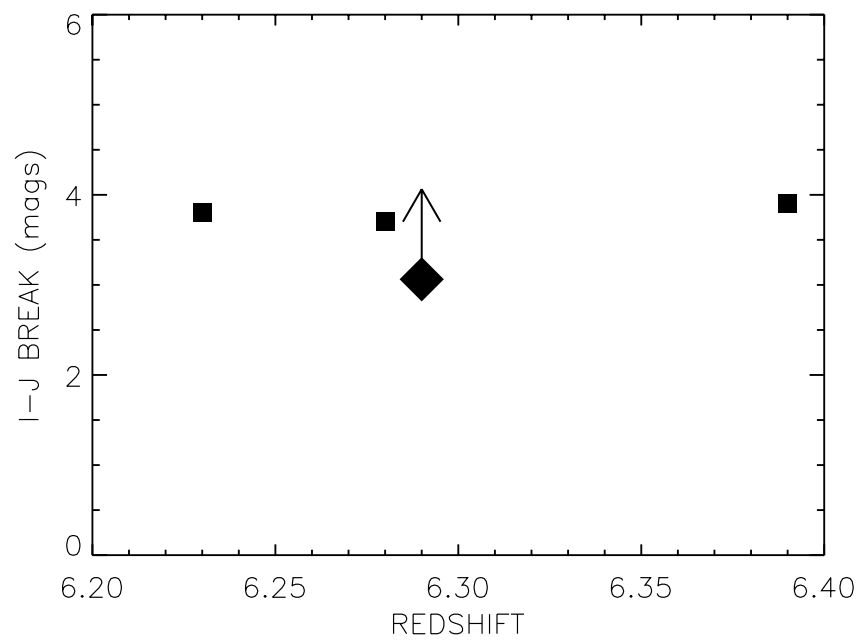

places a tight lower limit on the redshift of the source. Because the I-band data are consistent with a null detection, we cannot place any useful upper limit (better than $z \lesssim 8$ ) on the redshift of the source.

In order to obtain the redshift estimate, we fitted a power-law spectrum to the $J H K$ data, obtaining a spectral slope of $\beta=$ $0.3 \pm 0.6$, where $f_{\nu} \propto \nu^{-\beta}$; although the error bar is large, such a shallow spectral slope is likely a product of intrinsic variability in the source during the $J$-band observation, as discussed earlier. We then modulated this spectrum with the Ly $\alpha$ and Ly $\beta$ transmissions of the intergalactic medium measured by Songaila (2004) in high-redshift quasars. The resulting spectrum is shown for $z=$ 6.18 in Figure 2, where we also show the positions of the $I$ filter. In order to reduce the $I$-band flux to the observed value, we require $z>6.18$ at the $2 \sigma$ level and $z>6.37$ at the $1 \sigma$ level. The results are extremely sensitive to the adopted redshift. They are consistent with the redshift range of $z=6.30 \pm 0.07$ reported by Tagliaferri et al. (2005) based on similar observations with the Very Large Telescope and also with the spectroscopic redshift of $z=6.295 \pm$ 0.002 found by Kawai et al. (2006).

In Figure 3 we show the break strength between $R$ and $J$ and between $I$ and $J$, directly compared with the measured values in individual quasars at these redshifts. The quasar values are measured by comparing a power-law fit to the continuum in line-free regions of the quasar to the directly measured flux in the $I$ band (Songaila 2004). The $R$ band provides a weaker constraint than the $I$ band but would still place the GRB at $z>6.1$.

\section{DISCUSSION}

Observations of high-redshift GRBs can be used to infer the cosmological star formation history either through observations of the host galaxy or by translating the GRB rate to a star formation rate (Totani 1997). The most direct method is to assume that the rate of GRBs as a function of redshift is proportional to the rate of formation of high-mass stars and hence (assuming that the mass function is invariant) to the total star formation rate. In order to make such an interpretation, we need to calibrate the GRB rate versus the star formation history at lower redshifts, and it will always be subject to the assumption that the fraction of massive stars forming GRBs and the initial mass function of the stars remain invariant at the higher redshifts (e.g., Porciani \& Madau 2001; Lamb \& Reichart 2000; Bromm \& Loeb 2002; Natarajan et al. 2005).

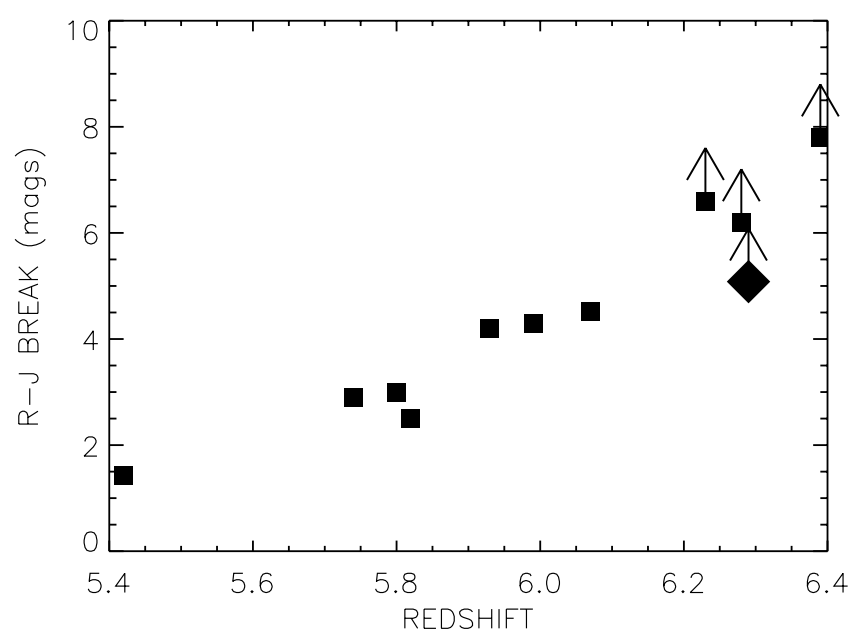

FIG. 3.-The $2 \sigma$ lower limits on the $I-J$ and $R-J$ break measured in the GRB (diamonds) are compared with directly measured values in high-redshift quasars (squares). 
TABLE 2

Lower Redshift Swift GRB Counterparts to GRB 050904

\begin{tabular}{|c|c|c|c|c|}
\hline GRB & Redshift & Peak Flux & $f_{z=6.29}$ & References \\
\hline $051111 \ldots \ldots \ldots \ldots \ldots \ldots \ldots \ldots \ldots$ & 1.549 & 2.50 & 0.25 & 1,2 \\
\hline 051109A... & 2.346 & 3.70 & 0.77 & 3,4 \\
\hline 050922C.. & 2.198 & 7.36 & 1.37 & 5,6 \\
\hline $050908 \ldots .$. & 3.350 & 0.70 & 0.27 & 7,8 \\
\hline 050820A.. & 2.612 & 2.50 & 0.63 & 9,10 \\
\hline $050802 \ldots$ & 1.710 & 2.65 & 0.32 & 11,12 \\
\hline $050730 \ldots$ & 3.969 & 0.57 & 0.28 & 13,14 \\
\hline $050603 \ldots$. & 2.821 & 27.6 & 7.89 & 15,16 \\
\hline $050505 \ldots .$. & 4.270 & 1.81 & 1.01 & 17,18 \\
\hline 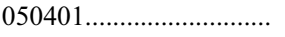 & 2.900 & 12.6 & 3.77 & 19,20 \\
\hline 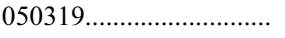 & 3.240 & 1.45 & 0.52 & 21,22 \\
\hline ..................... & 1.440 & 3.20 & 0.28 & 23,24 \\
\hline $050315 \ldots \ldots \ldots \ldots \ldots \ldots \ldots \ldots \ldots \ldots \ldots \ldots \ldots \ldots \ldots$ & 1.949 & 1.98 & 0.30 & 25,26 \\
\hline 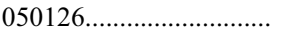 & 1.290 & 0.70 & 0.05 & 27,28 \\
\hline
\end{tabular}

Notes.-The sample is limited to Swift GRBs at $z>1$. The peak fluxes are in photons $\mathrm{cm}^{-2} \mathrm{~s}^{-1}$, measured in the $15-150 \mathrm{keV}$ band; they are taken from the Swift archive (http://swift.gsfc.nasa.gov/docs/swift/archive/grb_table.html). All but GRB 050126 would have been detected by Swift if placed at $z=6.29$.

ReFERENCES.-(1) Hill et al. 2005; (2) Krimm et al. 2005; (3) Quimby et al. 2005; (4) Fenimore et al. 2005; (5) Jakobsson et al. 2005; (6) Krimm et al. 2005; (7) Fugazza et al. 2005; (8) Sato et al. 2005; (9) Prochaska et al. 2005; (10) Cummings et al. 2005; (11) Fynbo et al. 2005; (12) Palmer et al. 2005; (13) Chen et al. 2005; (14) Markwardt et al. 2005; (15) Berger \& Becker 2005; (16) Fenimore et al. 2005; (17) Berger et al. 2005; (18) Hullinger et al. 2005; (19) Fynbo et al. 2005; (20) Sakamoto et al. 2005; (21) Fynbo et al. 2005; (22) Krimm et al. 2005; (23) Berger \& Mulchaey 2005; (24) Krimm et al. 2005; (25) Kelson \& Berger 2005; (26) Krimm et al. 2005; (27) Berger et al.2005; (28) Sato et al. 2005.

Prior to GRB 050904 twenty spectroscopic redshifts had been measured for Swift GRBs (excluding short/hard GRBs), of which fourteen lie beyond $z=1$. Thirteen of these fourteen would still have been detected above the Swift BAT threshold of 0.2 photons $\mathrm{cm}^{-2} \mathrm{~s}^{-1}$ if they had lain at $z=6.29$. This indicates that the selection effects, at least in the gamma rays, are not strong. Here we make the simple assumption that the 13 GRBs summarized in Table 2 that would have fluxes of about 0.2 photons $\mathrm{cm}^{-2} \mathrm{~s}^{-1}$ at $z=6.29$ represent the low-redshift counterparts of GRB 050904 detected over the same period of Swift observations, which indicates that the efficiency of detecting GRBs at $z=6.29$ is near unity, compared to GRBs at lower redshifts. For each GRB we give the redshift, the observed peak flux, and the value of the peak flux if the source had been at $z=6.29$.

We point out that this is, of course, a simplified analysis and that there are more selection effects than just the detection of the GRB itself. In particular, the success rate of detecting the optical/ NIR afterglow will be a function of redshift, as will the fraction of afterglows for which it is possible to measure the redshift, either from absorption or emission lines. This is a complicated endeavor requiring detailed Monte Carlo simulations of GRB afterglow searches to determine which GRBs with and without redshifts might plausibly have been identified if followed up in the same way as GRB 050904, and is beyond this simplified analysis. However, such an analysis is not justified at the present time, given the significant small-number uncertainties in the data and the desire for a larger sample of Swift events to use in bootstrapping the completeness estimate. Nevertheless, our measurement will provide, at the least, a lower limit to the star formation density, since we know that we are missing some GRBs.

Because of these complicated selection effects, which have discouraged all but the most bold (e.g., Blain \& Natarajan 2000) from using the actual redshifts measured from the optical after-

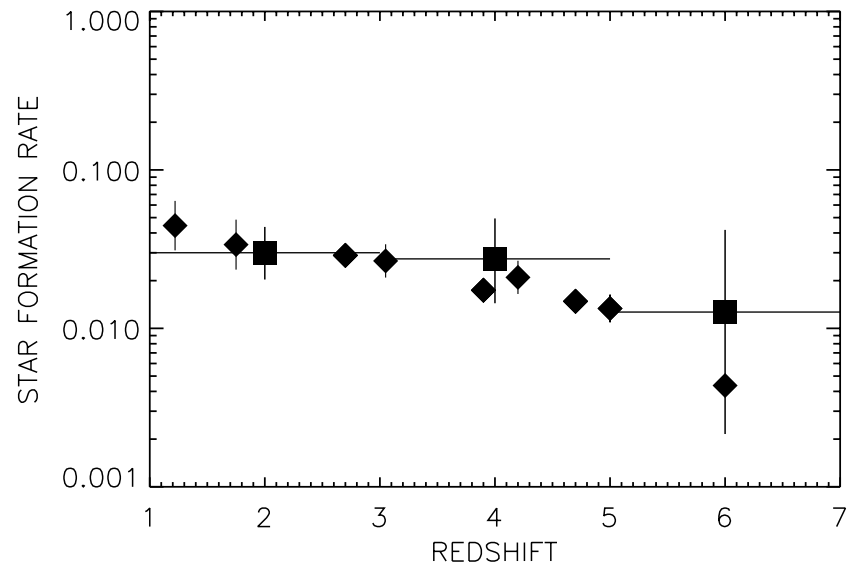

FIG. 4.-Star formation rate as a function of redshift in units of solar masses per $\mathrm{Mpc}^{3}$, taken from the compilation of Bunker et al. (2004), is shown by diamonds. The squares with $1 \sigma$ error bars show the corresponding determinations from the Swift GRBs for a normalizing constant of 0.0033 in the same units.

glow or host galaxy in determining the GRB rate as a function of redshift, most other attempts have relied on assuming that GRBs are standard candles (e.g., Totani 1999) or using empirical "pseudoredshift" indicators from the GRB itself (e.g., Firmani et al. 2004; Lloyd-Ronning et al. 2002), such as the luminosityvariability relation (Reichart et al. 2001). These methods buy a large sample size and better understood selection effects at the cost of uncertain (by at least a factor of 2!) redshifts. This results in a need to deconvolve the resulting rate distribution using the redshift errors, making it difficult to determine the true behavior of the GRB rate at high redshift.

The relation between the star formation history and the GRB rate has been derived by a number of authors (e.g., Porciani \& Madau 2001; Lamb \& Reichart 2000; Bromm \& Loeb 2002; Natarajan et al. 2005). At $z>1$, where the effects of the cosmological constant are negligible, the number of GRBs per unit redshift, $d N / d z$, in a given observing time interval is simply related to the star formation rate per unit comoving volume, $\psi$, by the function

$$
d N / d z=A \psi\left[(1+z)^{-2.5}-2(1+z)^{-3}+(1+z)^{-3.5}\right],
$$

where the normalizing factor, $A$, is assumed to be independent of $z$.

In Figure 4 we compare the shape of the star formation rate determined from the Swift GRBs with color-selected galaxy determinations of the star formation rate over the same redshift range taken from the paper of Bunker et al. (2004). We have set $A=0.005 M_{\odot} \mathrm{Mpc}^{-3}$ to match the observations at $z=3$. Within the wide uncertainties left by the small number of statistics, the current values cannot differentiate between the slow decline seen in the color-selected galaxies and a flat star formation rate with redshift. However, it is clear that as the sample size increases we should be able to make a valuable comparison. The GRB determinations are more powerful in some ways since they relate to all star formation, including that in lower luminosity galaxies than can be directly detected at the present time. This holds promise that future identifications of $z>6$ GRBs will enable a complete measurement of the star formation rate density at very high redshift.

\section{SUMMARY}

In the present paper we have described the observations of GRB 050904 with the MAGNUM telescope. These observations 
place a strong lower limit on the redshift of $z=6.18$ consistent with the spectroscopic redshift of $z=6.29$ measured by Kawai et al. (2006).

The most immediate result is that GRBs exist at $z>6$ and that they can be identified using a simple set of near-simultaneous optical and NIR observations. This presents the prospect of using the afterglows of high-redshift GRBs not only as lighthouses to illuminate the high-redshift universe (as is done for quasars today) but also as signposts to alert observers to the presence of the host galaxy, allowing deep follow-up observations to measure the Ly $\alpha$ flux. With the discovery of more high-redshift GRBs, it should be possible to form a useful sample for nailing down the epoch of reionization.

We also gave a simple discussion of the star formation rate history from $z=1$ to 7 based on the current Swift GRB observations showing that within the still broad uncertainties, the ob- servations point to a flat or a slowly declining star formation rate consistent with color-selected galaxy observations.

GRB 050904 is an exciting precursor to further high-redshift GRBs that should allow us to refine the star formation analysis and to study the properties of the intergalactic medium at these redshifts through color break and spectroscopic techniques.

We thank Elizabeth Stanway for providing the data for Figure 4 in tabular form. This work was supported by a Swift Guest Investigator grant (NNG05GF40G) and a Grant-in-Aid of the Center of Excellence Research (07CE2002) of the Ministry of Education, Science, Culture, and Sports of Japan. We thank the anonymous referee for helpful suggestions that improved this paper.
Andersen, M. I., et al. 2000, A\&A, 364, L54

Becker, R. H., et al. 2001, AJ, 122, 2850

Berger, E., \& Becker, G. 2005, GCN Circ. 3520, http:/gcn.gsfc.nasa.gov/gcn/ $\operatorname{gcn} 3 / 3520 . g \mathrm{cn} 3$

Berger, E., Cenko, S. B., \& Kulkarni, S. R. 2005a, GCN Circ. 3088, http:// gcn.gsfc.nasa.gov/gcn/gcn3/3088.gcn3

Berger, E., Cenko, S. B., Steidel, C., Reddy, N., \& Fox, D. B. 2005b, GCN Circ. 3368, http://gcn.gsfc.nasa.gov/gen/gcn3/3368.gcn3

Berger, E., \& Mulchaey, J. 2005, GCN Circ. 3122, http://gcn.gsfc.nasa.gov/ $\mathrm{gcn} / \mathrm{gcn} 3 / 3122 . \mathrm{gcn} 3$

Berger, E., et al. 2005c, ApJ, 634, 501

Blain, A. W., \& Natarajan, P. 2000, MNRAS, 312, L35

Bouwens, R. J., Illingworth, G. D., Blakeslee, J. P., \& Franx, M. 2006, ApJ, in press (astro-ph/0509641)

Bromm, V., \& Loeb, A. 2002, ApJ, 575, 111

Bunker, A. J., Stanway, E. R., Ellis, R. S., \& McMahon, R. G. 2004, MNRAS, 355,374

Chen, H.-W., Thompson, I., Prochaska, J. X., \& Bloom, J. 2005, GCN Circ. 3709, http://gcn.gsfc.nasa.gov/gcn/gcn3/3709.gcn3

Cummings, J., et al. 2005a, GCN Circ. 3910, http:/gcn.gsfc.nasa.gov/gen/ $\operatorname{gcn} 3 / 3910 . \mathrm{gcn} 3$

2005b, GCN Circ. 3835, http://gcn.gsfc.nasa.gov/gcn/gcn3/3835.gcn3

Fan, X., et al. 2001, AJ, 122, 2833

$$
\text { 2003, AJ, 125, } 1649
$$

2004, AJ, 128, 515

2005, AJ, 131, 1203

Fenimore, E., et al. 2005a, GCN Circ. 3512, http://gcn.gsfc.nasa.gov/gcn/gcn3/ 3512.gcn 3

2005b, GCN Circ. 4217, http://gcn.gsfc.nasa.gov/gen/gen3/4217.gcn3

Firmani, C., Avila-Reese, V., Ghisellini, G., \& Tutukov, A. V. 2004, ApJ, 611, 1033

Fox, D., \& Cenko, S. 2005, GCN Circ. 3912, http:/gcn.gsfc.nasa.gov/gen/ gen $3 / 3912 . g c n 3$

Fugazza, D., et al. 2005, GCN Circ. 3948, http:/gcn.gsfc.nasa.gov/gcn/gcn3/ 3948.gcn 3

Fynbo, J. P. U., Hjorth, J., Jensen, B. L., Jakobsson, P., Moller, P., \& Naranen, J. 2005a, GCN Circ. 3136, http://gcn.gsfc.nasa.gov/gcn/gcn3/3136.gcn3

Fynbo, J. P. U., et al. 2005b, GCN Circ. 3176, http://gcn.gsfc.nasa.gov/gcn/ gcn3/3176.gcn3

2005c, GCN Circ. 3749, http://gcn.gsfc.nasa.gov/gcn/gen3/3749.gcn3

Gunn, J. E., \& Peterson, B. A. 1965, ApJ, 142, 1633

Haislip, J., et al. 2005, Nature, submitted (astro-ph/0509660)

Hill, G., Prochaska, J. X., Fox, D., Schaefer, B., \& Reed, M. 2005, GCN Circ. 4255, http://gcn.gsfc.nasa.gov/gcn/gcn3/4255.gen3

Hu, E. M., \& Cowie, L. L. 2006, Nature, 440, 1145

Hu, E. M., Cowie, L. L., McMahon, R. G., Capak, P., Iwamuro, F., Kneib, J.-P., Maihara, T., \& Motohara, K. 2002, ApJ, 568, L75

Hullinger, D., et al. 2005, GCN Circ. 3364, http://gcn.gsfc.nasa.gov/gcn/gcn3/ 3364.gcn 3

Jakobsson, P., Fynbo, J. P. U., Paraficz, D., Telting, J., Jensen, B. L., Hjorth, J., \& Castro Ceron, J. M. 2005, GCN Circ. 4029, http://gcn.gsfc.nasa.gov/gen/ gen3/4029.gcn3
Kawai, N., et al. 2006, Nature, 440, 184

Kelson, D., \& Berger, E. 2005, GCN Circ. 3101, http://gcn.gsfc.nasa.gov/gen/ gen3/3101.gen3

Kobayashi, Y., Yoshii, Y., Peterson, B. A., Minezaki, T., Enya, K., Suganuma, M., \& Yamamuro, T. 1998, Proc. SPIE, 3354, 769

Kodaira, K., et al. 2003, PASJ, 55, L17

Krimm, H., et al. 2005a, GCN Circ. 4260, http://gcn.gsfc.nasa.gov/gen/gcn3/ 4260.gen3

2005b, GCN Circ. 4020, http://gcn.gsfc.nasa.gov/gcn/gcn3/4020.gcn3 2005c, GCN Circ. 3105, http://gcn.gsfc.nasa.gov/gen/gen $3 / 3105 . g c n 3$

\begin{tabular}{l} 
2005d, GCN Circ. 3134, http://gen.gsfc.nasa.gov/gen $/ g c n 3 / 3134 . g c n 3$ \\
\hline $.2005 \mathrm{e}$, GCN Circ. 3119, http://gen.gsfc.nasa.gov/gen $/ g \ln 3 / 3119 . g c n 3$
\end{tabular}

Kurk, J. D., Cimatti, A., di Serego Alighieri, S., Vernet, J., Daddi, E., Ferrara, A., \& Ciardi, B. 2004, A\&A, 422, L13

Lamb, D. Q., \& Reichart, D. E. 2000, ApJ, 536, 1

Lipkin, Y. M., et al. 2004, ApJ, 606, 381

Lloyd-Ronning, N. M., Fryer, C. L., \& Ramirez-Ruiz, E. 2002, ApJ, 574, 554

Markwardt, C. B., et al. 2005, GCN Circ. 3715, http://gcn.gsfc.nasa.gov/gen/ gcn3/3715.gcn3

Natarajan, P., Albanna, B., Hjorth, J., Ramirez-Ruiz, E., Tanvir, N., \& Wijers, R. 2005, MNRAS, 364, L8

Palmer, D., et al. 2005, GCN Circ. 3737, http://gcn.gsfc.nasa.gov/gcn/gcn3/ 3737.gcn 3

Porciani, C., \& Madau, P. 2001, ApJ, 548, 522

Prochaska, J. X., Bloom, J. S., Wright, J. T., Butler, R. P., Chen, H. W., Vogt, S. S., \& Marcy, G. W. 2005, GCN Circ. 3833, http://gen.gsfc.nasa.gov/gen/gen3/ 3833.gen3

Quimby, R., Fox, D., Hoeflich, P., Roman, B., \& Wheeler, J. C. 2005, GCN Circ. 4221, http://gcn.gsfc.nasa.gov/gcn/gcn3/4221.gcn3

Reichart, D. E., Lamb, D. Q., Fenimore, E. E., Ramirez-Ruiz, E., Cline, T. L., \& Hurley, K. 2001, ApJ, 552, 57

Rhoads, J. E., et al. 2004, ApJ, 611, 59

Sakamoto, T., et al. 2005, GCN Circ. 3173, http:/gcn.gsfc.nasa.gov/gcn/gcn3/ 3173.gen3

Sato, G., et al. 2005a, GCN Circ. 3951, http://gcn.gsfc.nasa.gov/gen/gcn3/ 3951.gcn3

2005b, GCN Circ. 2987, http://gcn.gsfc.nasa.gov/gen/gen3/2987.gcn3

Smith, J. A., et al. 2002, AJ, 123, 2121

Songaila, A. 2004, AJ, 127, 2598

Stern, D., Yost, S. A., Eckart, M. E., Harrison, F. A., Helfand, D. J., Djorgovski, S. G., Malhotra, S., \& Rhoads, J. E. 2005, ApJ, 619, 12

Tagliaferri, G., et al. 2005, A\&A, 443, L1

Taniguchi, Y., et al. 2005, PASJ, 57, 165

Totani, T. 1997, ApJ, 486, L71

1999, ApJ, 511, 41

Wyithe, J. S. B., \& Loeb, A. 2003, ApJ, 586, 693

Yoshii, Y. 2002, in New Trends in Theoretical and Observational Cosmology, ed. K. Sato \& T. Shiromizu (Tokyo: Universal Academy Press), 235 